\title{
Communicable Diseases Report, NSW, November-December 2007
}

\section{Communicable Diseases Branch, NSW Department of Health}

For updated information, including data and facts on specific diseases, visit www.health.nsw.gov.au and click on Infectious Diseases.

Tables 1 and 2 and Figure 1 show reports of communicable diseases received through to the end of November and December 2007 in NSW.

\section{Meningococcal disease}

In NSW, meningococcal disease was notified in eight people in November and 12 people in December. In total, 108 cases were notified in the 12 months to December 2007, including five deaths. Of the 2007 cases, nine were due to serogroup $C$ meningococcal bacteria and 73 were due to serogroup B. In 2006, 102 cases were notified including six deaths.

\section{Enteric Diseases}

In November, NSW public health units investigated 58 outbreaks of gastroenteritis, including two suspected to be foodborne and 56 suspected to be caused by person-toperson spread. The two suspected foodborne outbreaks involved groups of 11 and three people, respectively, at different restaurants; no specimens were available for testing from either outbreak. Among the 56 suspected person-to-person outbreaks, 39 were in age care facilities and affected 657 people, 11 were in hospitals that affected 85 people, five were in child-care centres and affected 45 children, and one was in a school and affected 16 children. For comparison, 70 outbreaks were reported in October 2007 and 29 were reported in November 2006.

In December, NSW public health units investigated 10 outbreaks of gastroenteritis, including three suspected to be foodborne outbreaks and seven suspected to be caused by person-to-person spread. The three foodborne outbreaks were caused by salmonellosis and consumption of undercooked or raw eggs was suspected to be a possible source of infection. No links between the outbreaks were identified. Of the seven outbreaks of gastroenteritis, four were in aged care facilities and affected 31 people, two were in hospitals and affected eight people, and one was in a child-care centre and affected six children.

\section{Increase in reported cases of shiga toxin-producing Escherichia coli}

Shiga toxin-producing E. coli (STEC) are bacteria that can cause serious gastrointestinal disease characterised by diarrhoea, which in some cases can be bloody. In a small proportion of cases STEC can progress to haemolytic uraemic syndrome (HUS), which results in kidney failure, bleeding and anaemia. Infections tend to increase in the warmer months. ${ }^{1}$

In November, NSW public health units were notified of seven cases of STEC (3 serotype O157, 1 serotype O111, and 1 serotype O26) and three cases of HUS. The ages of the cases ranged from 2 to 71 years. Seven cases were male and three female. Seven cases resided in the Hunter New England Area, two in South East Sydney Illawarra Area and one in the Greater Southern Area. The HUS cases were all children aged 2 to 5 years; STEC (untyped) was also identified in one of the HUS cases. Although 10 cases within a month appears unusually high, the total number of cases in 2007 (16 STEC and 11 HUS cases) is similar to previous years. Interviews with the cases or their carers did not identify a likely common source of infection.

In December, NSW public health units were notified of seven STEC (1 serotype O157, 1 serotype O111, 1 serotype $\mathrm{O} 55$ and 4 of unknown serotype) and two HUS cases. The age of cases ranged from 11 months to 75 years. Six were female and three male. All HUS cases were adults aged over 40 years. This number of STEC and HUS cases reported in December 2007 is slightly higher than the number seen in December 2006.

STEC infection can be transmitted through:

- eating contaminated food (undercooked hamburgers, unwashed salad, fruit, vegetables and unpasteurised milk or milk products)

- drinking or swimming in contaminated water

- person-to-person contact; for example, contact with faeces of an infected child when changing a nappy

- contact with infected animals.,3

The most important ways to prevent infection with STEC and other foodborne diseases are to:

- cook hamburgers and sausages thoroughly to at least $71^{\circ} \mathrm{C}$. Although colour alone is not necessarily a good 
indicator, do not eat hamburgers or sausages if there is any pink meat inside

- wash hands well after handling raw meat

- use different knives and cutting boards for raw meat preparation and other food preparation

- wash raw vegetables and fruits thoroughly

- refrigerate perishable food until ready to eat

- wash hands well after touching animals or their faeces.

For more information see: http:/www.health.nsw.gov. au/infect/pdf/stec_cdfs.pdf.

\section{Listeriosis}

In December, four cases of listeriosis were reported in NSW, two male and two female. The age of cases ranged from 28 to 75 years. Cases reported eating a range of highrisk foods; however, no common source of infection was identified. One case was a pregnant woman; she and her babies recovered.

Listeriosis is usually caused by ingestion of contaminated food and has been associated with consumption of undercooked or raw meat, runny eggs, soft cheeses, unpasteurised milk and pre-prepared and unwashed vegetables. Those at highest risk are unborn babies, the elderly, immune compromised people and pregnant women. Listeriosis is particularly important for pregnant women as the infection can cause foetal death. ${ }^{4}$

\section{Increase in reported cases of cryptosporidiosis}

There were 153 cases of cryptosporidiosis reported as having their onset date in November and 84 in December in NSW. This compares with 34 cases in October. The highest rate of infection was in children under five years of age (see: http://www.health.nsw.gov.au/data/diseases/ cryptosporidiosis.html) and in rural areas.
Cryptosporidiosis is a diarrhoeal disease caused by a parasitic infection of the intestine. The most common symptoms include diarrhoea, abdominal cramps and sometimes fever, nausea and vomiting. Symptoms may last a few weeks in some people. ${ }^{5}$

Public health officers interviewed cases who report a range of possible risk factors, including contact with farm animals, drinking untreated water and swimming.

In the past, large outbreaks in NSW have been caused by people swimming in contaminated pools. ${ }^{6}$ Pools can easily be contaminated by infectious swimmers. To keep pools free from contamination, people should not swim in a pool or spa until at least two weeks after they have completely recovered from a diarrhoeal illness.

\section{References}

1. Tarr PI, Gordon CA, Chandler WL. Shiga-toxin-producing Escherichia coli and haemolytic uraemic syndrome. Lancet 2005; 365: 1073-86.

2. Heymann D, ed. Control of communicable diseases manual, 18th edn. Washington: American Public Health Association, 2004:160-4.

3. Razzaq S. Hemolytic Uremic Syndrome: An Emerging Health Risk. Am Fam Physician 2006; 74: 991-7.

4. Heymann D, ed. Control of communicable diseases manual, 18th edn. Washington: American Public Health Association, 2004:309-315.

5. Heymann D, ed. Control of communicable diseases manual, 18th edn. Washington: American Public Health Association, 2004:138-41.

6. Black M, McAnulty J. The investigation of an outbreak of cryptosporidiosis in New South Wales in 2005. N S W Public Health Bull 2006; 17: 76-9. doi:10.1071/NB06018 
Figure 1. Reports of selected communicable diseases, NSW, January 2002 to December 2007, by month of onset.

Preliminary data: case counts in recent months may increase because of reporting delays.

Laboratory-confirmed cases only, except for measles, meningococcal disease and pertussis.

BFV, Barmah Forest virus infections; RRV, Ross River virus infections; Lab Conf, laboratory confirmed;

Men $\mathrm{Gp} C$ and $\mathrm{Gp} B$, meningococcal disease due to serogroup $C$ and serogroup B infection;

other/unk, other or unknown serogroups.

NB: multiple series in graphs are stacked, except gastroenteritis outbreaks.

NB: Outbreaks are more likely to be reported by nursing homes and hospitals than by other institutions.

\begin{tabular}{|lc|}
\hline \multicolumn{2}{|l|}{ NSW Population } \\
Male & $50 \%$ \\
$<5$ y & $7 \%$ \\
$5-24 y$ & $27 \%$ \\
$25-64 y$ & $53 \%$ \\
$65+y$ & $13 \%$ \\
Rural & $46 \%$ \\
\hline
\end{tabular}
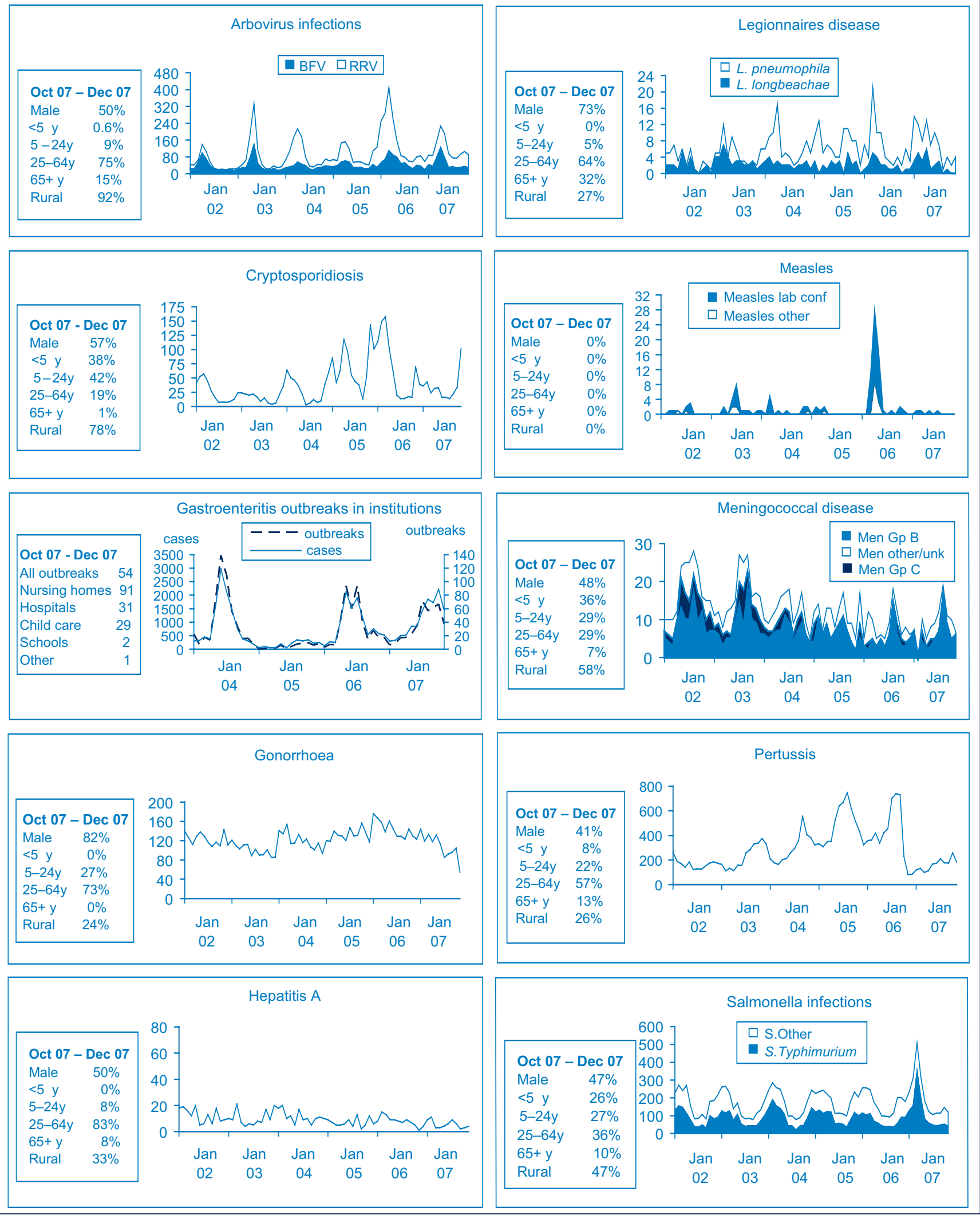
Table 1. Reports of notifiable conditions received in November 2007 by Area Health Services

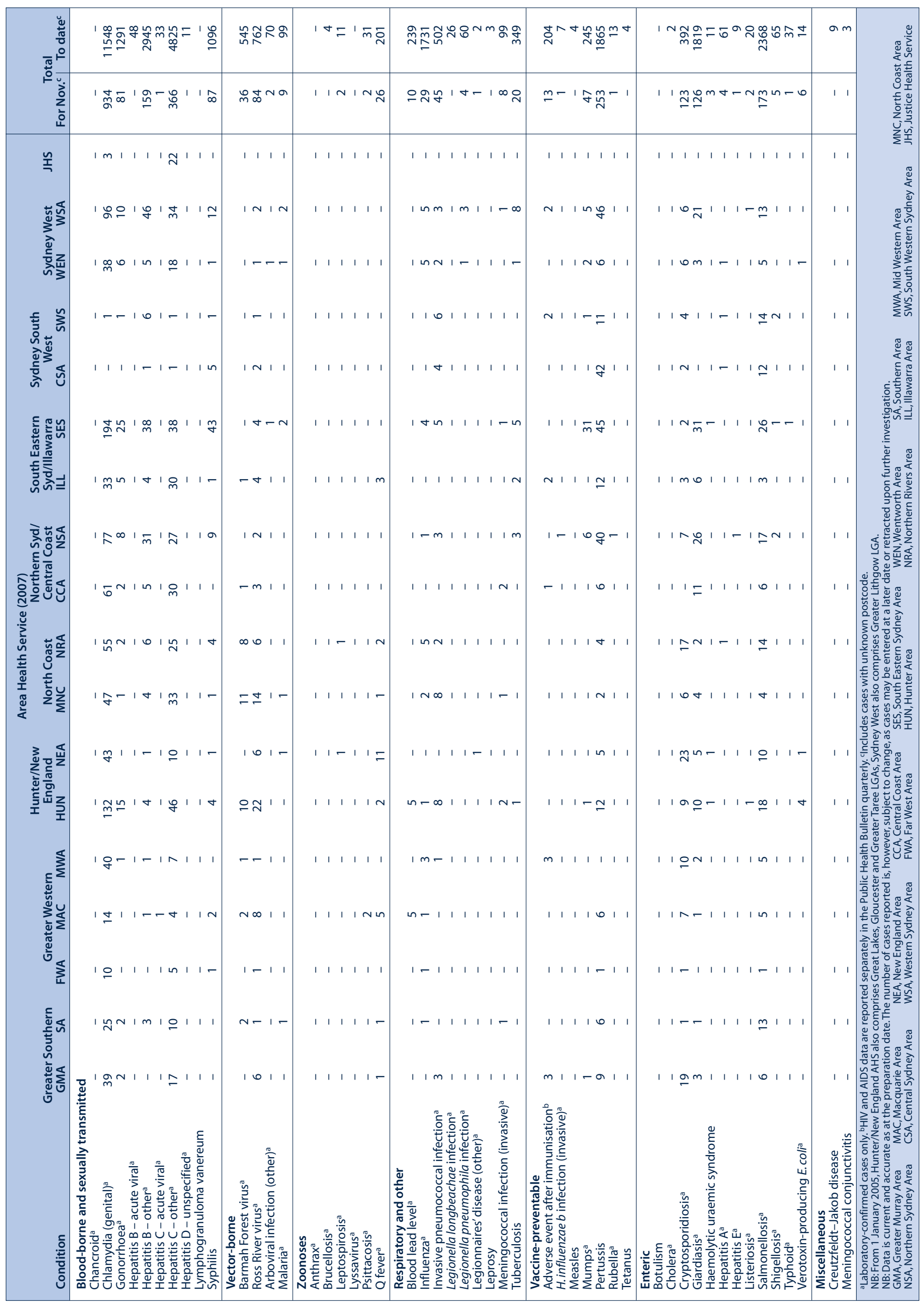


Table 2. Reports of notifiable conditions received in December 2007 by Area Health Services

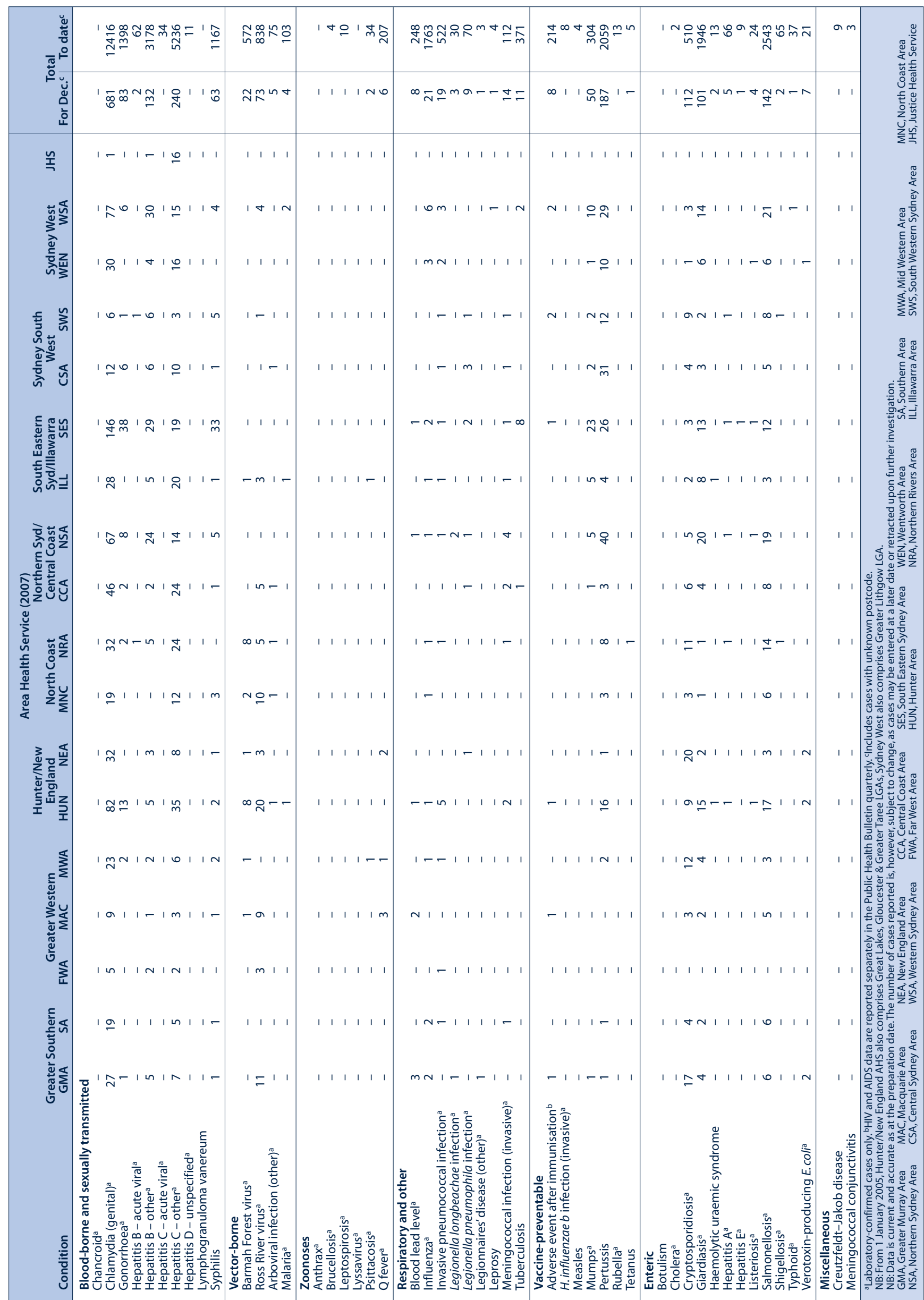

